\title{
Is it more accurate to measure the absolute or the relative change of new high or ultrasensitive troponin assays to diagnose acute myocardial infarction?
}

\author{
Reviewed by: Rohit Mohindra, MASc, MD*; Carine Haggar, MD ${ }^{\dagger}$
}

\begin{abstract}
Clinical question
In emergency department patients with symptoms suggestive of acute myocardial infarction, is the absolute or relative change of cardiac troponin assays (either high sensitivity or ultrasenstive) more accurate in diagnosing acute myocardial infarction at 1 to 2 hours postpresentation?

Article chosen

Rechlin T, Ifran A, Twerenbold R, et al. Utility of absolute and relative changes in cardiac troponin concentrations in the early diagnosis of acute myocardial infarction. Circulation 2011;124:136-45.

Objective

To evaluate the accuracy of new troponin assays in diagnosing acute myocardial events.
\end{abstract}

Keywords: acute myocardial infarction, diagnostic accuracy, emergency department, high-sensitivity troponin

\section{BACKGROUND}

New highly sensitive or ultrasensitive assays can detect cardiac troponin (cTn) levels below the 99th percentile for the normal population with a coefficient of variation (as a measure of precision) $\leq 10 \%$. This allows for measurement of previously undetectable cTn levels. Potential benefits could include earlier intervention or discharge of patients. ${ }^{1,2}$ The potential downside would be increased sensitivity at a cost of lowered specificity, resulting in admission of many patients not having suffered an acute coronary syndrome (ACS). Current guidelines specify that one value above the 99th percentile is significant for myocardial necrosis but do not specify if absolute or relative change is preferred when interpreting serial cTn values. ${ }^{1,3}$ In the article reviewed, Reichlin and colleagues evaluated the diagnostic accuracy of absolute and relative cTn changes (from highly sensitive and ultrasensitive assays) in the early diagnosis of acute myocardial infarction (AMI).

\section{STUDY DESIGN}

This was a prospective observational cohort substudy of the Advantageous Predictors of Acute Coronary Syndromes Evaluation (APACE) study. Funding was received from the Swiss National Science Foundation, Swiss Heart Foundation, Abbott, Roche, Siemens, and the University Hospital Basel. The study was conducted in Basel, Switzerland, between April 2006 and September 2009. Inclusion criteria were emergency department (ED) patients with chest pain onset or peak within the last 12 hours. Patients with terminal kidney failure or a diagnosis of ST segment elevation myocardial infarction (STEMI) were excluded. Ethics board approval and informed consent were obtained.

All patients were assessed and treated at the discretion of the attending physician. The initial clinical assessment for each patient included clinical history, physical examination, 12-lead electrocardiography (ECG), ECG monitoring, pulse oximetry, blood tests, and chest

From the *FRCPC Emergency Medicine Residency Program, McGill University, and the †Department of Emergency Medicine, McGill University Health Centre, Montreal, QC.

Correspondence to: Dr. Rohit Mohindra, McGill University FRCPC Emergency Medicine Residency Program, Royal Victoria Hospital, Room A4.62, 687 Pine Avenue West, Montreal, QC H3H 1A1; rohit.mohindra@mail.mcgill.ca.

This article has been peer reviewed. 
radiography. Standard (nonindex) cTn levels were measured at presentation and after 6 to 9 hours or as clinically indicated.

The index tests of interest were the Roche highsensitivity cardiac troponin $\mathrm{T}$ (hs-cTnT) and the Siemens ultrasensitive cardiac troponin I (cTnI-ultra) assays. ${ }^{4}$ Samples were taken at presentation and at 1 and/or 2 hours postpresentation and were frozen for future assessment.

The reference test for diagnosis of AMI was as adjudicated by two independent cardiologists blinded only to hs-cTnT or cTnI-ultra results. AMI was defined as per current guidelines. ${ }^{5}$

Area under the curve (AUC) measurements were derived. Statistical significance was reported using two-tailed comparison tests with a significance threshold of $p<0.05$ and confidence intervals.

\section{RESULTS}

After excluding patients with STEMI and patients without the index test available, 836 patients were included in the study (Figure 1). AMI was diagnosed in 108 patients, who tended to be older, with previous embolic disease, worse renal function, less heart failure, and higher baseline cTn.

In patients with AMI, early ( 1 or 2 hours postpresentation) absolute and relative changes in the hscTnT and cTnI-ultra were significantly higher than in patients without a final diagnosis of AMI. The absolute change in AUC was higher compared to relative changes (Table 1). Optimal cutoff values for absolute changes within 2 hours were $0.007 \mu \mathrm{g} / \mathrm{L}$ for hs-cTnT and $0.020 \mu \mathrm{g} / \mathrm{L}$ for cTnI-ultra. If combined with baseline troponin levels, 1- and 2-hour cTn values demonstrated improved accuracy. For hs-cTnT, baseline cTn levels had an AUC of 0.94 and improved to $0.98(p=0.001)$ when combined with 1 -hour cTn values. A small subset of 305 patients had cTn values available at 1, 2, and 6 hours postpresentation (Table 2). Accuracy at 1 hour did not vary significantly from accuracy at 6 hours postpresentation. Positive and negative predictive values are shown in Table 3.

Subgroup analysis demonstrated similar trends with male and female patients, patients $>70$ years old, and those with renal dysfunction or heart failure. The accuracy of the diagnosis was unaffected by time from symptoms onset or baseline cTn levels.

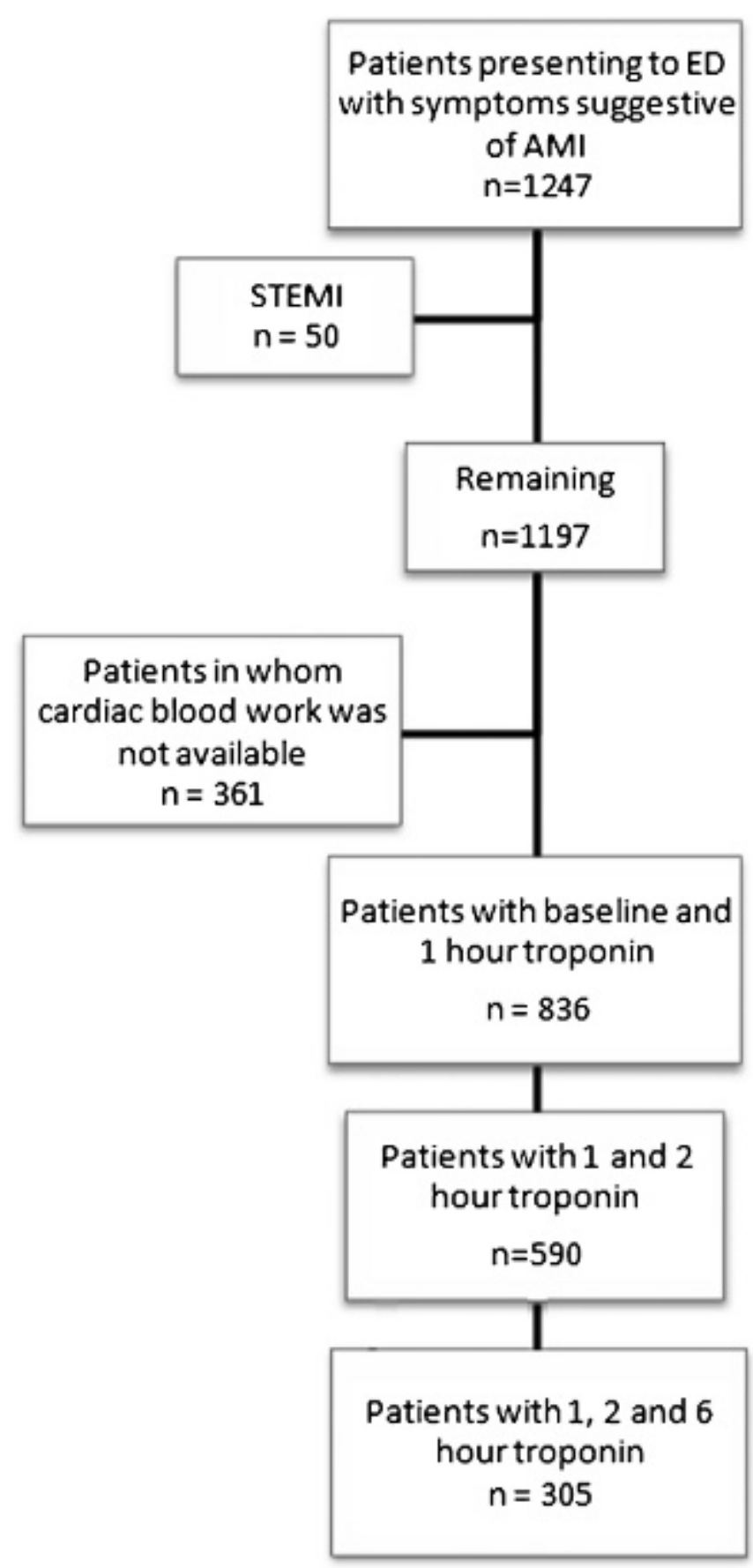

Figure 1. Distribution of patients after consecutive sampling and enrolment in the study. A total of 836 patients were enrolled, from a pool of 1,247 possible participants. AMI = acute myocardial infarction, ED = emergency department; STEMI = ST segment elevation myocardial infarction.

\section{STUDY CONCLUSIONS}

Absolute cTn changes were superior to relative $c T n$ changes in diagnosing AMI for both the hs-c'TnT and cTnI-ultra assays. The diagnostic superiority of 


\begin{tabular}{|c|c|c|c|c|}
\hline & \multicolumn{2}{|c|}{ Absolute change $(95 \% \mathrm{Cl})$} & \multicolumn{2}{|c|}{ Relative change $(95 \% \mathrm{Cl})$} \\
\hline & hs-cTnT & cTnl-ultra & hs-cTnT & cTnl-ultra \\
\hline 1 hour postpresentation & $0.93(0.90-0.96)$ & $0.94(0.91-0.97)$ & $0.66(0.60-0.72)$ & $0.64(0.59-0.69)$ \\
\hline 2 hours postpresentation & $0.95(0.92-0.98)$ & $0.95(0.91-0.99)$ & $0.76(0.70-0.83)$ & $0.72(0.66-0.79)$ \\
\hline
\end{tabular}

absolute cTn changes was independent of the initial cTn baseline value and consistent within patient subgroups. Diagnostic accuracies for AMI as provided by absolute changes (within 1 and 2 hours) were comparable to those measured within 6 hours.

\section{EVALUATION}

\section{Are the results of the study valid?}

The authors accurately described a relevant patient population, defined as ED patients with potential cardiac chest pain or angina pectoris. An appropriate reference standard was used, and adjudicators were blinded to assay results.

This study took place at a large, urban tertiary centre, and referral bias may cause overestimation of diagnostic accuracy. ${ }^{6,7}$ Controlled studies of cTn measurements often perform better than real-world data would suggest. ${ }^{5}$ The study did not explicitly address the influence of funding sources on the study. The potential for performance, design, and reporting bias needs to be considered when interpreting the results. $^{8}$

The superior performance of absolute change could be related to a delayed later presentation time of patients with ACS. These patients may already be approaching a plateau phase for $\mathrm{c}$ Tn release, and the

Table 2. Area under the curve for cTn samples drawn at 1, 2, and 6 hours

\begin{tabular}{l|cc|}
\hline & $\begin{array}{c}\text { Area under the curve-absolute } \\
\text { change }\end{array}$ \\
\cline { 2 - 3 } & hs-cTnT & cTnl-ultra \\
\hline 1 hour postpresentation & 0.91 & 0.91 \\
2 hours postpresentation & 0.96 & 0.94 \\
6 hours postpresentation & 0.95 & 0.95 \\
\hline $\begin{array}{l}\text { cTn = cardiac troponin; cTnl-ultra = ultrasensitive cardiac troponin I assay; hs-cTnT = } \\
\text { high-sensitivity cardiac troponin T assay. } \\
\text { Results are available only for the absolute change. }\end{array}$ \\
\hline
\end{tabular}

relative cTn changes are likely low regardless of test performance. ${ }^{9,10}$

\section{Are the results of the study useful?}

From the study results, it is not clear if this early diagnosis will lead to better patient outcomes. A recently published observational cohort study suggests that this approach can predict mortality at 1 year. ${ }^{11}$ The effect on patient-centred outcomes is not clear.

Although the AUC is commonly used, there are both mathematical and rhetorical arguments for two tests having the same AUC but different test properties. ${ }^{12,13}$ The claim of a negative predictive value of $100 \%$ may overemphasize diagnostic certainty. Reporting likelihood ratios for multiple thresholds may provide a more intuitive comparison. ${ }^{14}$

The high negative predictive value potentially allows an earlier exclusion of AMI in patients presenting with chest pain. This may represent substantial implications for departmental flow. ${ }^{15-18}$ Many patients with ACS but not suffering an AMI may still require admission. The relatively poor test specificity will lead to longer ED stays for follow-up testing and/or consultations in patients not suffering from ACS. $2,9,19$

The authors reported no significant difference in diagnostic accuracy between baseline and cTn drawn at 1,2 , and 6 hours postpresentation. If such high sensitivities and accuracies are validated by further study, this could allow for more rapid exclusion of AMI in patients with chest pain.

\section{Will the results change practice?}

As high-sensitivity c $T$ n becomes ubiquitous in EDs, results as presented by Reichlin and colleagues could allow for diagnosis of AMI earlier than seen with the current standard cTn measurement (baseline and 6 hours postpresentation). Use of absolute c'Tn changes will result in more accurate diagnosis compared to relative changes. The ability to have earlier diagnosis 
Table 3. Positive predictive value and negative predictive value for absolute and relative changes in hs-cTnT and cTnl-ultra measurements in patients presenting with chest pain

\begin{tabular}{|c|c|c|c|c|c|c|c|c|}
\hline & \multicolumn{4}{|c|}{ Absolute change } & \multicolumn{4}{|c|}{ Relative change } \\
\hline & \multicolumn{2}{|c|}{ hs-cTnT } & \multicolumn{2}{|c|}{ cTnl-ultra } & \multicolumn{2}{|c|}{ hs-cTnT } & \multicolumn{2}{|c|}{ cTnl-ultra } \\
\hline & PPV & NPV & PPV & NPV & PPV & NPV & PPV & NPV \\
\hline 1 hour postpresentation & 66 & 97 & 64 & 98 & 27 & 91 & 19 & 91 \\
\hline 2 hours postpresentation & 64 & 98 & 58 & 99 & 35 & 94 & 32 & 93 \\
\hline
\end{tabular}

of AMI could prompt the initiation of appropriate therapies sooner and assist with timely ED disposition planning. Validation in additional cohorts, with specific assessment of the impact of lower specificities on clinical practice, is required before incorporation into current practice.

Acknowledgement: Dr. Farooq Khan is acknowledged for assistance with the original analysis of the article.

Competing interests: None declared.

\section{REFERENCES}

1. Thygesen K, Alpert JS, White HD. Universal definition of myocardial infarction. 7 Am Coll Cardiol 2007;50:2173-95, doi:10.1016/j.jacc.2007.09.011.

2. Omland T. Sensitive cardiac troponin assays: sense and sensibility. Eur Heart 7 2012;33:944-6, doi:10.1093/eurheartj/ ehs009.

3. Antman EM, Anbe DT, Armstrong PW, et al. The management of patients with ST-elevation myocardial infarction: a report of the American College of Cardiology/American Heart Association Task Force. Circulation 2004;110:e82-292, doi:10.1161/01.CIR.0000134791.68010.FA.

4. Reichlin T, Hochholzer W, Bassetti S, et al. Early diagnosis of myocardial infarction with sensitive cardiac troponin assays. $N$ Engl 7 Med 2009;361:858-67, doi:10.1056/ NEJMoa0900428.

5. Thygesen K, Mair J, Katus H, et al. Recommendations for the use of cardiac troponin measurement in acute cardiac care. Eur Heart 7 2010;31:2197-204, doi:10.1093/eurheartj/ ehq251.

6. Whiting P, Rutjes AWS, Reitsma JB, et al. Sources of variation and bias in studies of diagnostic accuracy: a systematic review. Ann Intern Med 2004;140:189-202, doi:10.7326/0003-4819-140-3-200402030-00010.

7. Rutjes AWS, Reitsma JB, Di Nisio M, et al. Evidence of bias and variation in diagnostic accuracy studies. Can Med Assoc 7 2006;174:469-76, doi:10.1503/cmaj.050090.

8. Bekelman JE. Scope and impact of financial conflicts of interest in biomedical research: a systematic review. $7 A M A$ 2003;289:454-65, doi:10.1001/jama.289.4.454.
9. Mueller M, Biener M, Vafaie M, et al. Absolute and relative kinetic changes of high-sensitivity cardiac troponin $\mathrm{T}$ in acute coronary syndrome and in patients with increased troponin in the absence of acute coronary syndrome. Clin Chem 2012;58:209-18, doi:10.1373/clinchem.2011.171827.

10. Apple FS. Edging closer to early optimal patient management with high-sensitivity cardiac troponin assay. Circulation 2011; 124:127-9, doi:10.1161/CIRCULATIONAHA.111.038851.

11. Aldous S, Richards M, Cullen L. Diagnostic and prognostic utility of early measurement with high-sensitivity troponin $\mathrm{T}$ assay in patients presenting with chest pain. Can Med Assoc 7 2012;184:260-8, doi:10.1503/cmaj.110773.

12. Hilden J. What properties should an overall measure of test performance possess? Clin Chem 2005;51:471; author reply 471-2, doi:10.1373/clinchem.2004.041376.

13. Reichlin T, Irfan A, Twerenbold R, et al. Utility of absolute and relative changes in cardiac troponin concentrations in the early diagnosis of acute myocardial infarction. Circulation 2011; 124:136-45, doi:10.1161/CIRCULATIONAHA.111.023937.

14. Furukawa TA, Strauss S, Bucher HC, Guyatt G. Diagnostic tests. In: Guyatt G, Rennie D, Meade MO, Cook DJ, editors. Users' guides to the medical literature: a manual for evidence-based clinical practice. 2nd ed. New York: McGrawHill; 2008. p. 419-38.

15. Twerenbold R, Jaffe A, Reichlin T, et al. High-sensitive troponin $\mathrm{T}$ measurements: what do we gain and what are the challenges? Eur Heart 7 2012;33:579-86, doi:10.1093/ eurheartj/ehr492.

16. Mangleson FIJ, Cullen L, Scott AC. The evolution of chest pain pathways. Crit Patbw Cardiol 2011;10:69-75, doi:10. 1097/HPC.0b013e3182215b48.

17. Reiter M, Twerenbold R, Reichlin T, et al. Early diagnosis of acute myocardial infarction in the elderly using more sensitive cardiac troponin assays. Eur Heart 7 2011;32:137989, doi:10.1093/eurheartj/ehr033.

18. Reiter M, Twerenbold R, Reichlin T, et al. Early diagnosis of acute myocardial infarction in patients with pre-existing coronary artery disease using more sensitive cardiac troponin assays. Eur Heart 7 2012;33:988-97, doi:10.1093/eurheartj/ ehr376.

19. Vasikaran SD, Macdonald SPJ, Sikaris K. High-sensitivity cardiac troponin assays for risk stratification and for the diagnosis of acute myocardial infarction. Ann Clin Biochem 2012;49(Pt 3):209-10, doi:10.1258/acb.2012.012058. 